04

\title{
Влияние параметров разряда на генерацию ионов дейтерия в плазме сильноточной импульсной вакуумной дуги с композиционным катодом из дейтерида циркония
}

\author{
(С) А.Г. Николаев, ${ }^{1}$ Е.М. Окс, ${ }^{1,2}$ В.П. Фролова, ${ }^{1}$ Г.Ю. Юшков, ${ }^{1}$ Д.Л. Шмелев, ${ }^{3}$ \\ И.В. Уйманов, ${ }^{3}$ C.A. Баренгольц ${ }^{4,5}$ \\ ${ }^{1}$ Институт сильноточной электроники СО РАН, \\ 634055 Томск, Россия \\ ${ }^{2}$ Томский государственный университет систем управления и радиоэлектроники, \\ 634050 Томск, Россия \\ ${ }^{3}$ Институт электрофизики УрО РАН, \\ 620016 Екатеринбург, Россия \\ ${ }^{4}$ Институт общей фризики им. А.М. Прохорова РАН, \\ 119991, Москва, Россия \\ ${ }^{5}$ Физический институт им. П.Н. Лебедева РАН, \\ 119991 Москва, Россия \\ e-mail: nik@opee.hcei.tsc.ru
}

(Поступило в Редакцию 31 мая 2016 г. В окончательной редакции 25 октября 2016 г.)

\begin{abstract}
Проведено исследование масс-зарядового состава пучка ионов, извлеченных из плазмы вакуумной дуги с катодом из дейтерида циркония при различной длительности импульса тока дуги (полуширина на полувысоте), составляющей 2, 4, 7 и $17 \mu \mathrm{s}$. Установлено, что доля ионов дейтерия в плазме вакуумной дуги повышается с ростом тока, а при токах порядка $1 \mathrm{kA}$ эта зависимость выходит на насыщение. При долевом содержании атомов дейтерия в катоде на уровне $40 \%$, доля ионов дейтерия в плазме вакуумной дуги может достигать более 80\%. Дана теоретическая интерпретация полученных экспериментальных результатов. Показано, что основным источником ионов дейтерия в микросекундном вакуумном дуговом разряде являются катодные пятна. Для количественной оценки содержания ионов дейтерия в дуговой плазме разработана модель десорбции дейтерия при функционировании катодных пятен.
\end{abstract}

DOI: 10.21883/JTF.2017.05.44439.1912

\section{Введение}

Вакуумный дуговой разряд с композиционным катодом из дейтерида циркония применяется для генерации импульсных нейтронных потоков в вакуумных нейтронных трубках [1]. По сути нейтронная трубка представляет собой миниатюрный вакуумный дуговой ионный источник, выполненный в виде отпаянного прибора с длительностью импульса порядка $1 \mu \mathrm{s}$. Достигнутая доля атомарных ионов дейтерия в плазме вакуумной дуги определяет эффективность работы такого устройства. При функционировании катодного пятна вакуумной дуги на поверхности дейтерида циркония наряду с ионами дейтерия образуются и ионы циркония, а также относительно небольшое количество примесей, включающих в себя ионы газов, адсорбированных поверхностью катода из остаточной атмосферы, а также ионы распыленных в разряде материалов электродов разрядной системы.

Дальнейшее повышение интенсивности генерации потоков нейтронов в подобных устройствах возможно в результате увеличения среднего тока ускоренных ионов дейтерия, которое, в свою очередь, может быть достигнуто увеличением тока дугового разряда или его длительности. Ранее нами были проведены исследования по получению ионов дейтерия в плазме квазистационарной вакуумной дуги с током порядка 100 А и длительностью импульса в сотни микросекунд [2,3]. Анализ полученных в этих работах результатов обусловил необходимость изучения процессов генерации ионов дейтерия в сильноточном режиме горения дуги (килоамперный диапазон токов) более короткой длительности (единицы микросекунд), направленных на достижение максимальной доли ионов дейтерия в плазме дугового разряда. О целесообразности реализации сильноточного режима дуги короткой длительности свидетельствует тот факт, что именно для этого режима горения дуги характерна высокая эффективность ионизации, обеспечивающая достижение максимального зарядового состояния ионов в плазме [4].

\section{Влияние длительности импульса и амплитуды тока на содержание ионов дейтерия в дуговой плазме}

Эксперименты проводились в лаборатории плазменных источников Института сильноточной электроники СО РАН с использованием экспериментального стенда вакуумного дугового источника Mevva-V.Ru. Конструктивные особенности и принцип действия источника 


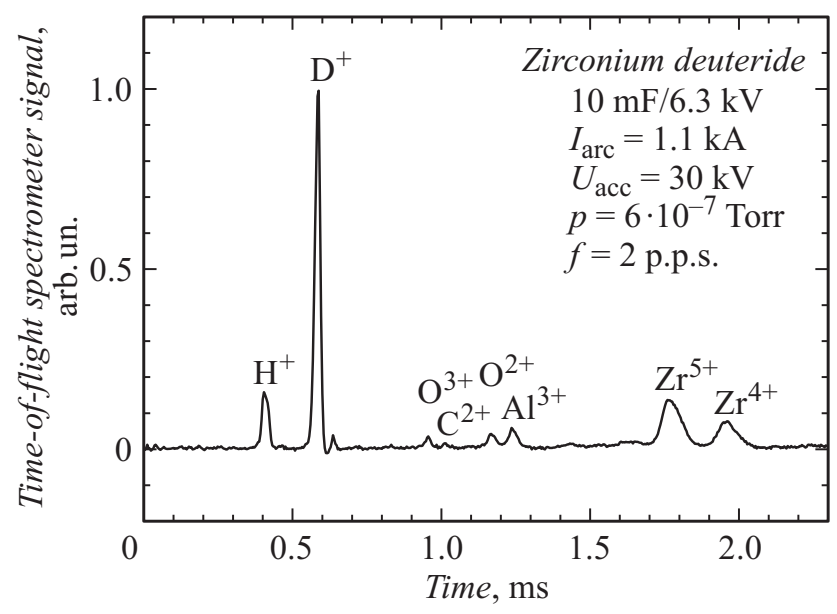

Pис. 1. Масс-зарядовый спектр ионного пучка, извлеченного из плазмы вакуумной дуги с катодом из дейтерида циркония. Ток дуги $-1.1 \mathrm{kA}$.

подробно изложены в [5]. Функционирование источника основано на генерации плазмы в катодных пятнах вакуумной дуги и отборе с развитой поверхности этой плазмы ионов материала катода. Формирование ионного пучка осуществлялось при приложении между эмиссионным и ускоряющим электродами напряжения величиной до $60 \mathrm{kV}$. Оценка плотности тока ионного пучка осуществлялась цилиндром Фарадея, входящая апертура которого площадью $10 \mathrm{~cm}^{2}$ была много меньше площади сечения пучка. При этом по форме импульса тока на цилиндр Фарадея можно было отслеживать временную зависимость полного ионного тока пучка. Масс-зарядовый состав плазмы разряда определялся по измерению состава ионного пучка. Для этой цели применялся времяпролетный спектрометр с пролетной базой $1 \mathrm{~m}$, конструкция и основные параметры которого приведены в [6]. Ионный источник размещался на вакуумной камере объемом $0.6 \mathrm{~m}^{3}$ с откачкой криогенным насосом со скоростью 3200 1/s до предельного остаточного давления $3 \cdot 10^{-7}$ Torr. Импульс тока дуги формировался в результате разряда на межэлектродный промежуток конденсатора. Использовались низкоиндуктивные (менее $20 \mathrm{nH}$ ) конденсаторы емкостью $0.22,1$ и $10 \mu \mathrm{F}$, а также конденсатор емкостью $20 \mu \mathrm{F}$ с индуктивностью порядка $300 \mathrm{nH}$. Длительность импульса тока дуги (полуширина на полувысоте) при этом составляла 2, 4, 7 и $17 \mu$ s соответственно. Амплитуда импульса тока дуги определялась напряжением зарядки конденсатора и в экспериментах варьировала от 100 А до $3 \mathrm{kA}$. Основные эксперименты были проведены с частотой повторения импульсов тока 2 p.p.s., при которой интегральный нагрев катода был несуществен.

В экспериментах использовался катод из дейтерида циркония с атомарным содержанием дейтерия 40 at.\%. Катод был выполнен в виде шайбы с внешним диамет- ром $23.5 \mathrm{~mm}$, внутренним диаметром $9.2 \mathrm{~mm}$ и толщиной $1.8 \mathrm{~mm}$.

Типичный спектр масс-зарядового состава пучка ионов, извлеченных из плазмы вакуумной дуги с катодом из дейтерида циркония, представлен на рис. 1. Как видно из спектра, ионы дейтерия превалируют в извлеченном из плазмы ионном пучке, а следовательно, и в самой плазме вакуумной дуги.

В экспериментах эволюция масс-зарядового спектра ионного пучка отслеживалась в течение импульса тока с временным интервалом $2 \mu \mathrm{s}$. Каждый спектр усреднялся не менее, чем по 100 измерениям. Одновременно осциллографировались импульсы тока дуги и тока ионного пучка. По спектрам и осциллограмме тока пучка рассчитывались долевые соотношения для основных компонентов ионного пучка (дейтерия и циркония), а также суммарная доля всех примесей. Характерные осциллограммы импульсов тока разряда, тока ионного пучка на цилиндр Фарадея и зависимость от времени его основных компонентов и примесей приведены на рис. 2.

Обратим внимание на следующие особенности. Импульс тока ионного пучка имеет большую длительность и временную задержку относительно импульса тока дуги. Импульсы компонентов ионного пучка (дейтерия, циркония, примесей) сдвинуты друг относительно друга и их длительности также различны. Наблюдаемые особенности связаны с различными значениями направленной скорости в плазме ионов различных масс, определяющей время достижения ионами эмиссионной плазменной границы, удаленной от катодной области на расстояние $15 \mathrm{~cm}$ [7]. Именно поэтому для количественного определения масс-зарядового состава ионов измеренный ток каждой ионной фракции интегрировался по всему импульсу. Как показали измерения (рис. 1 и 2), доля ионов примесей, включающих, главным образом, ионы водорода, не превышала $10 \%$ от тока пучка. При этом доля примесных ионов оставалась практически

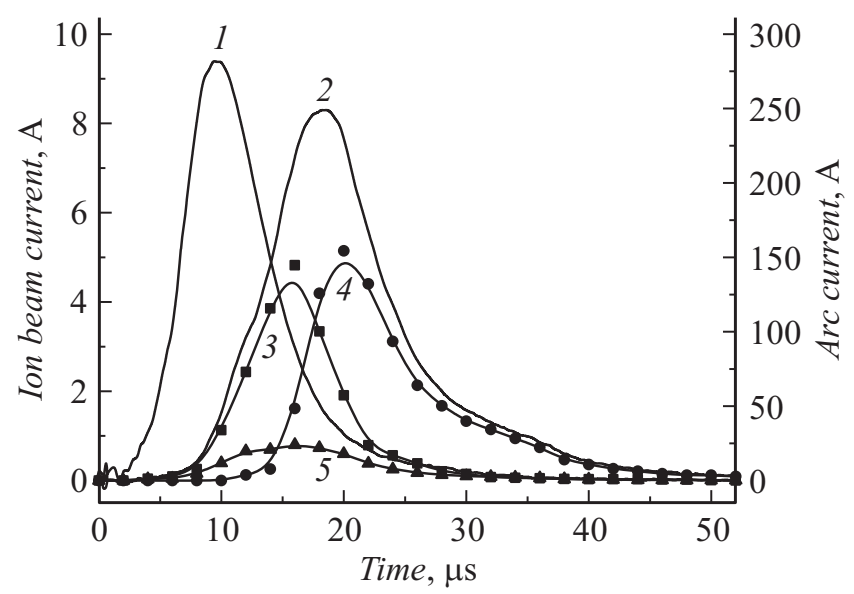

Рис. 2. Осциллограммы импульсов: 1 - тока разряда (правая шкала), 2 - тока ионного пучка на цилиндр Фарадея, и зависимость от времени тока его основных фракций: 3 дейтерия, 4 - циркония, 5 - примесей. 


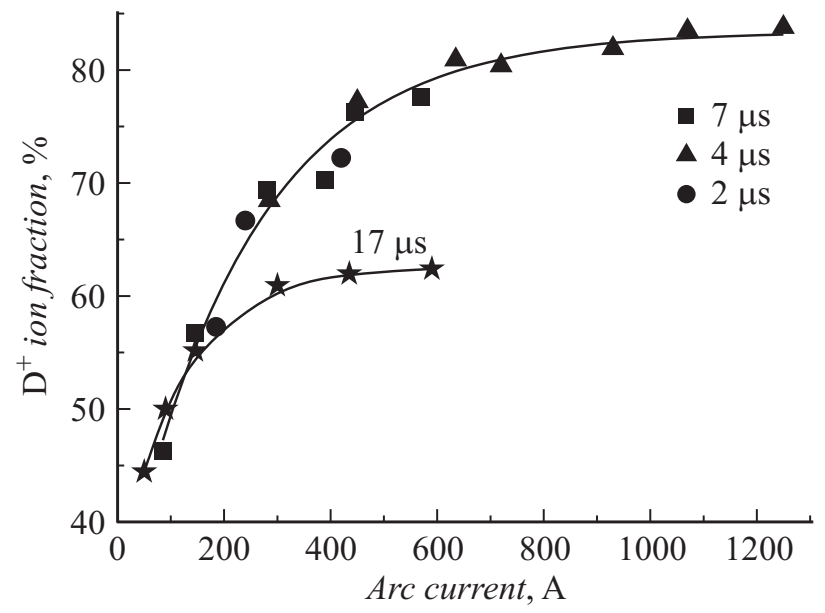

Pис. 3. Зависимость интегральной доли ионов дейтерия в полном количестве ионов материала катода в импульсе тока ионного пучка от тока дугового разряда. Ускоряющее напряжение $30 \mathrm{kV}$, давление $5 \cdot 10^{-7}$ Torr.

постоянной и при оценках влияния параметров дугового разряда на долю ионов дейтерия не учитывалась.

Влияние амплитуды тока и длительности импульса тока дуги на интегральную долю ионов дейтерия в полном количестве ионов материала катода (дейтерида циркония) в импульсе тока ионного пучка иллюстрируется данными, представленными на рис. 3.

Обращает на себя внимание следующее:

- доля ионов дейтерия в общем количестве основных компонентов ионного пучка (дейтерия и циркония), а следовательно, и в плазме вакуумной дуги повышается с ростом тока дугового разряда;

- зависимость доли ионов дейтерия от тока разряда стремится к насыщению;

- увеличение длительности импульса тока дуги от 2 до $7 \mu \mathrm{s}$ не оказывает влияния на зависимость количества ионов дейтерия от тока дуги;

- при большей длительности импульса тока дуги $(17 \mu \mathrm{s})$ характер зависимости от тока дуги не изменяется, но насыщение достигается при меньших значениях доли ионов дейтерия в плазме;

- при долевом содержании молекул дейтерия в дейтериде циркония 40\%, максимальная доля ионов дейтерия в плазме вакуумной дуги достигает $83 \%$ для импульсов короткой длительности и $62 \%$ для импульса большей длительности.

\section{Моделирование процесса десорбции дейтерия в катодных пятнах вакуумной дуги}

В настоящее время практически „adhoc“ полагается, что основными поставщиками ионов дейтерия в плазму дугового источника являются катодные пятна (КП) [8].
В теоретическом плане доступны лишь весьма приблизительные и небесспорные оценки, сделанные на основе расчета глубины проникновения температурного фронта в катод за время горения разряда и предположения о полной десорбции дейтерия из нагретого объема $\mathrm{ZrD}_{x}$ [9]. Высокая температура катода в КП, достигающая нескольких тысяч градусов Кельвина, является серьезным аргументом в пользу этой концепции. Однако оценим возможность ионизации дейтерия, десорбированного с поверхности катода, в объеме разряда. Для этого рассмотрим цилиндрический объем (с сечением, соответствующим площади катода $\left.S_{c} \sim 5 \mathrm{~cm}^{2}\right)$, заполненный однородной плазмой с усредненными параметрами. Для оценки возьмем максимальный поток ионов дейтерия. Как следует из рис. 3, на ионы дейтерия в максимуме приходится $\sim 83 \%$ от общего числа ионов, т. е. дейтронов больше, чем ионов циркония в $\sim 4.9$ раза. Тогда максимальный поток дейтерия $G$ оценим, как $G=4.9 n_{i} v_{i} \approx 3.4 \cdot 10^{20} \mathrm{~cm}^{-2} \mathrm{~s}^{-1}, v_{i}=1.54 \cdot 10^{6} \mathrm{~cm} / \mathrm{s}$ средняя скорость ионов циркония в вакуумной дуге [7], средняя плотность ионов циркония в объеме разряда $n_{i}=(\mathrm{Jg}) /\left(v_{i} m_{i}\right) \approx 0.45 \cdot 10^{14} \mathrm{~cm}^{-3}$, где $g=53 \mu \mathrm{g} / \mathrm{C}-$ удельная эрозия циркония [10], $J \approx 200 \mathrm{~A} / \mathrm{cm}^{2}$ - плотность тока при токе дуги $1 \mathrm{kA}$. Следует заметить, что мы использовали максимальную величину удельной эрозии циркония, известную из литературы, потому что здесь мы проводим оценку максимального потока ионов. Тогда за одну микросекунду горения разряда $\left(t_{\mathrm{arc}}\right)$ при данном потоке $G$ проходит порядка $G S_{c} t_{\mathrm{arc}} \approx 1.7 \cdot 10^{15}$ ионов дейтерия. Поверхностная плотность адсорбированных молекул дейтерия в одном монослое на поверхности цирконии может достигать $10^{15} \mathrm{~cm}^{-2}$ [11]. Таким образом, одна лишь десорбция дейтерия из поверхностных состояний (без диффузии дейтерия из объема) способна обеспечить необходимое количество дейтерия. Оценим теперь возможности диссоциации и ионизации. Будем учитывать лишь прямую диссоциацию и ионизацию дейтерия электронным ударом, тогда имеем

$$
\begin{gathered}
V_{d} \frac{\partial C_{0}}{\partial z}=-A_{0}\left(T_{e}\right) C_{0} n_{e}, \\
V_{d} \frac{\partial C_{1}}{\partial z}=2 A_{0}\left(T_{e}\right) C_{0} n_{e}-A_{1}\left(T_{e}\right) C_{1} n_{e}, \\
C_{2}=1-2 C_{0}-C_{1}, \quad n_{e}=2.5 n_{i}+\frac{G C_{2}}{V_{d}}, \\
\left.C_{0}\right|_{z=0}=0.5,\left.\quad C_{1}\right|_{z=0}=0,
\end{gathered}
$$

где $C_{0}, C_{1}, C_{2}$ - относительные концентрации молекул, атомов и ионов дейтерия соответственно, $n_{e}$ - плотность электронов в предположении квазинейтральности, $A\left(T_{e}\right)$ - скорости соответствующих реакций [12]. После численного интегрирования системы уравнений получаем, что при электронной температуре $T_{e}$ в плазме разряда, равной $5 \mathrm{eV}$, на расстоянии $5 \mathrm{~cm}$ от катода $\left(V_{d}=5 \cdot 10^{5} \mathrm{~cm} / \mathrm{s}\right) C_{2} \approx 1.5 \cdot 10^{-4}$, т. е. только $0.015 \%$ от необходимого потока дейтерия будет ионизовано. При $T_{e}=15 \mathrm{eV}, C_{2} \approx 4 \cdot 10^{-3}$, что тоже пренебрежимо мало 
Результаты численного моделирования

\begin{tabular}{c|c|c|c|c|c|c|c|c|c}
\hline$j_{0} \cdot 10^{-8}, \mathrm{~A} / \mathrm{cm}^{2}$ & $I_{c}, \mathrm{~A}$ & $\langle T\rangle, \mathrm{K}$ & $t_{c}, \mathrm{~ns}$ & $\eta_{1}, \%$ & $h_{c}, \mu \mathrm{m}$ & $r_{c}, \mu \mathrm{m}$ & $r_{p}, \mu \mathrm{m}$ & $\Delta V_{p} \cdot 10^{18}, \mathrm{~m}^{3}$ & $\eta_{2}, \%$ \\
\hline 1.0 & 3 & 4517 & 25 & 97.9 & 1.16 & 2.71 & 1.91 & 5.41 & 85.3 \\
1.0 & 5 & 4944 & 28 & 96.0 & 1.21 & 3.1 & 2.34 & 8.78 \\
1.0 & 8 & 5249 & 30 & 93.4 & 1.22 & 3.65 & 2.75 & 12.7 \\
1.5 & 3 & 4897 & 18 & 87.4 & 1.04 & 2.03 & 1.61 & 3.63 & 81.1 \\
1.5 & 5 & 5233 & 20 & 87.7 & 1.08 & 2.51 & 2.03 & 5.75 & 82.3 \\
1.5 & 8 & 5698 & 23 & 80.8 & 1.16 & 3.60 & 2.45 & 9.23 \\
2.0 & 3 & 5346 & 13 & 84.6 & 0.89 & 1.85 & 1.4 & 2.35 & 80.7 \\
2.0 & 5 & 5566 & 16 & 83.8 & 1.03 & 2.3 & 1.81 & 4.42 & 81.7 \\
2.0 & 8 & 6093 & 18 & 83.4 & 1.07 & 2.8 & 2.2 & 7.02 & 79.8
\end{tabular}

по сравнению с регистрируемым потоком дейтронов. Таким образом, наблюдаемый поток дейтронов не может быть обеспечен ионизацией дейтерия в объеме разряда. С другой стороны, согласно модели [13], ионизация в плазменной струе катодного пятна способна обеспечить требуемый поток дейтронов. Так, при превышении потоком дейтерия потока ионов циркония в 5 раз на расстоянии $30 \mu \mathrm{m}$ от пятна уже происходит $\sim 93 \%$ ионизация атомов дейтерия. Таким образом, основным источником ионов дейтерия в микросекундном вакуумном дуговом разряде, скорее всего, являются катодные пятна.

Для получения оценок количества десорбированного дейтерия из КП в [9] использовалось представление о стационарном характере функционирования единого катодного пятна в течение всего разряда. Однако в работах Кесаева [14] было убедительно показано, что КП вакуумной дуги состоит из отдельных ячеек. Данное положение легло в основу эктонной модели [15], которая предполагает к тому же циклический характер функционирования отдельных ячеек КП, имеющих характерный микронный пространственный масштаб и время жизни в несколько десятков наносекунд. При этом ток, переносимый отдельной ячейкой, ограничен и не превосходит $\sim 10 \mathrm{~A}$, а общий ток дуги складывается из токов отдельных ячеек и тем самым определяет количество одновременно функционирующих ячеек. Тогда в рамках эктонной модели КП процессы десорбции дейтерия и формирование элементного состава плазмы в элементарной ячейке КП будут преимущественно определять эти параметры и для дугового источника в целом.

Для анализа десорбционных характеристик единичной ячейки КП нами разработана модель конвективной термодиффузии дейтерия и его десорбции в плазму дугового разряда, горящего на $\mathrm{ZrD}_{0.67}$-катоде [16]. Тепломассоперенос и перенос заряда в Zr-матрице рассчитывается в рамках 2D осесимметричной полуэмпирической гидродинамической модели образования кратера [17]. Анализ процессов переноса дейтерия в $\mathrm{ZrD}_{x}$-катоде в КП и проведенные оценки позволили сделать вывод, что электроперенос дейтерия и перенос, вызванный градиентами напряжений, возникающими при неоднородном нагреве катода, пренебрежимо малы по сравнению с диффузией и термопереносом и поэтому в модели не учитываются. Таким образом, в рамках предложенного подхода моделируются выделение и перенос тепла в $\mathrm{ZrD}_{0.67}$-катоде, которые приводит к образованию расплава, развитие гидродинамического возмущения поверхности расплава, вызванного давлением плазмы КП на катод и приводящего к образованию кратера и формированию жидкометаллических струй, а также десорбция с поверхности катода, конвекция (совместно с $\mathrm{ZrD}_{0.67}$-расплавом) и термодиффузия дейтерия в катоде. Коэффициент диффузии дейтерия в $\beta$-фазе $\mathrm{ZrD}_{0.67}$ в широком диапазоне температур рассчитывается по закону Аррениуса путем экстраполяции экспериментальных данных [18] для водорода с учетом изотопического эффекта $D_{D}=D_{H} / 1.5[19]$.

На рис. 4 показан профиль кратера и распределение дейтерия в $\mathrm{ZrD}_{0.67}$-катоде к моменту времени $t_{c}=20 \mathrm{~ns}$. Данное время, при котором формируется струя длиной $\sim 1 / 3$ радиуса кратера, в модели рассматривается как время инициирования новой ячейки КП за счет электрического взрыва этой струи. И соответственно $t_{c}$ определяет время жизни (окончание протекания тока) моделируемой ячейки. В таблице представлены результаты моделирования при различных токах ячейки $I_{c}$, который предполагается постоянным в течении $t_{c}$, и начальной плотности тока $j_{0}$, которая уменьшается на $20-30 \%$ по мере развития поверхности кратера. В третьей колонке таблицы представлена средняя температура поверхности дна кратера $\langle T\rangle$ - температура, усредненная по поверхности впадины $z<0$ (рис. 4) и по времени. Доля ионов дейтерия в плазме $\eta_{1}=N_{D} /\left(N_{D}+N_{\mathrm{Zr}}\right) \cdot 100 \%$, полученная в рамках численного расчета количества десорбированного дейтерия $N_{D}$ и количества испарившегося циркония $N_{\mathrm{Zr}}$ за время $t_{c}$ при образовании кратеpa, представлена в пятой колонке таблицы. Величины $N_{D}$ и $N_{\mathrm{Zr}}$ рассчитывались путем интегрирования по поверхности катода и времени диффузионного потока атомов дейтерия и ленгмюровского потока испарения атомов циркония (с данными по давлению насыщенных паров для чистого $\mathrm{Zr}$ из [20]) соответственно. Отметим, что в рамках предложенной модели самосогласованного расчета характеристик плазмы КП и соответственно 


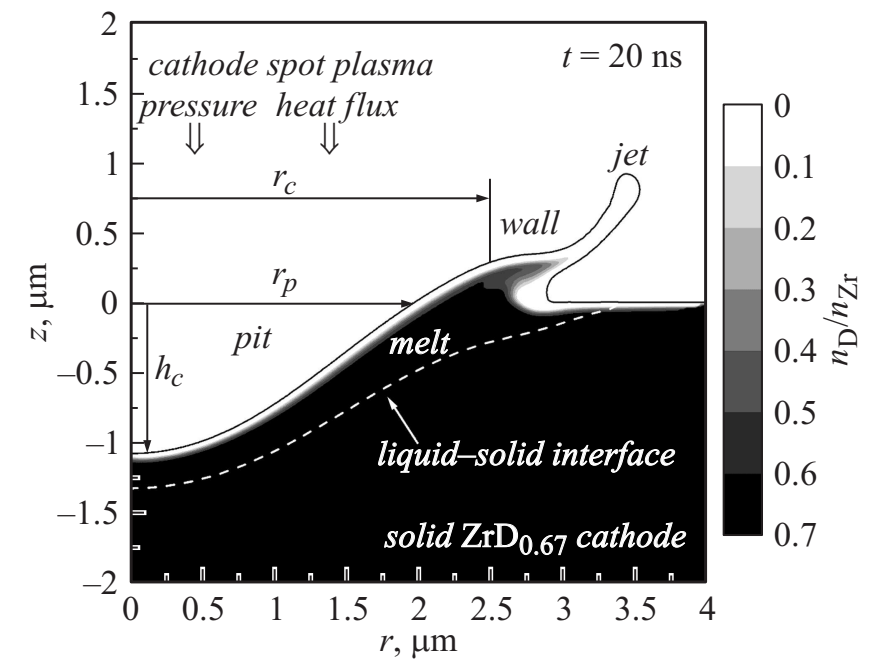

Рис. 4. Профиль кратера и распределение дейтерия в $\mathrm{ZrD}_{0.67}$-катоде к моменту времени $t_{c}=20 \mathrm{~ns}$. Параметры модели: $I_{c}=5 \mathrm{~A}, j_{0}=1.5 \cdot 10^{8} \mathrm{~A} / \mathrm{cm}^{2}$.

обратных потоков ионов дейтерия и циркония из плазмы КП на катод не производится. Поэтому предложенный способ определения композиционного состава плазмы КП корректен, если предполагать, что обратные потоки с одинаковым коэффициентом пропорциональны прямым потокам атомов дейтерия и циркония с поверхности катода в плазму.

Как видно из таблицы, хорошее согласие с нашим экспериментом в случае коротких импульсов наблюдается при параметрах моделирования, когда начальная плотность тока в ячейке $j_{0} \geq 1.5 \cdot 10^{8} \mathrm{~A} / \mathrm{cm}^{2}$. В этих случаях средняя температура поверхности кратера больше $\sim 5300 \mathrm{~K}$. При меньших плотностях тока в ячейке и соответственно меньших температурах поверхности кратера количество испарившихся атомов $\mathrm{Zr}$ как минимум на 2 порядка меньше количества десорбируемых атомов дейтерия за время жизни единичной ячейки. Разумно полагать, что в этом случае композиционный состав плазмы КП существенно отличался бы от наблюдаемого нами в эксперименте. Это обстоятельство может косвенно указывать на то, что даже средняя температура поверхности катода под плотной плазмой КП в вакуумной дуге превосходит $\sim 5000 \mathrm{~K}$. Отметим, что данный параметр имеет фундаментальное значение для теории КП вакуумной дуги и на сегодняшний день недоступен для прямого экспериментального изучения. Однако увеличение плотности тока в ячейке оказывает влияние не только на повышение средней температуры поверхности кратера. В рамках рассматриваемой модели с ростом плотности тока растет и давление плазмы на катод, что приводит к меньшим временам жизни ячейки.

На основании результатов проведенного моделирования картина процесса десорбции дейтерия при образовании кратера выглядит следующим образом. Рост температуры поверхности катода $\geq \sim 1100 \mathrm{~K}$ приводит к быстрой десорбции поверхностных состояний за время $<1 \mathrm{~ns}$ вне зависимости от использования в модели порядка кинетики десорбции (от второго до нулевого порядка) и различных экспериментальных данных по энергии активации десорбции. Учет процессов стимулированной десорбции дейтерия за счет обратного потока частиц из плазмы на катод только усиливает это обстоятельство. Однако доля десорбированного дейтерия на этой стадии незначительна по сравнению с его количеством, которое десорбируется за все время функционирования ячейки $t_{c}$. Поэтому лимитирующим фактором, преимущественно определяющим десорбцию дейтерия за время $t_{c}$, является его перенос в катоде из объема к поверхности. При этом существенную роль в процессе десорбции играет движение расплава. Так как скорость его движения максимальна в приповерхностных слоях, формирование вала кратера и струй происходит путем натекания приповерхностных, почти полностью десорбированных слоев (рис. 4). С другой стороны, выдавливание десорбированного расплава из центра на периферию приводит к выходу на поверхность во впадине кратера менее десорбированных слоев, так что диффузионный приповерхностный слой остается тонким и обеспечивается большой градиент концентрации дейтерия вблизи наиболее нагретой поверхности кратера. Моделирование десорбции дейтерия с учетом последующей фазы остывания $\left(t>t_{c}\right)$, которая начинается после прекращения протекания тока, позволило предложить другой метод определения элементного состава плазмы КП с дейтерированным катодом. Оценочно объем полностью десорбированного расплава можно определить по объему впадины, так как в силу условия несжимаемости расплава объем вала и струй равен объему впадины. А вал и струи практически полностью десорбируются в процессе их формирования (рис. 4). В колонках 6-8 таблицы представлены пространственные параметры кратеров, а в колонке 9 вытесненный объем $\Delta V_{p}$, рассчитанные при моделировании. Тогда количество десорбированного дейтерия $N_{D}$ можно оценить как $N_{D} \approx \Delta V_{p} \cdot 0.67 n_{\mathrm{Zr}}$, где $n_{\mathrm{Zr}}$ - концентрация атомов циркония в катоде. А количество испарившегося циркония $N_{\mathrm{Zr}}$ в ячейке с током $I_{c}$ за время $t_{c}$ можно определить по удельной эрозии $g$ : $N_{\mathrm{Zr}}=g I_{c} t_{c}$. Рассчитанные данным способом процентные содержания дейтерия в плазме элементарной ячейки $\eta_{2}=\Delta V_{p} \cdot 0.67 n_{\mathrm{Zr}} /\left(\Delta V_{p} \cdot 0.67 n_{\mathrm{Zr}}+g I_{c} t_{c}\right) \cdot 100 \%$ представлены в последней колонке таблицы. Эти значения хорошо согласуются с полученными нами экспериментальными значениями для коротких разрядов при всех начальных параметрах модели. При расчете $\eta_{2}$ нами использовалось значение $g=53 \mu \mathrm{g} / \mathrm{C}$ для чистого циркония [20]. Однако необходимо отметить, что в случае $\mathrm{ZrD}_{x} g$ может существенно отличаться и даже нелинейным образом зависеть от степени дейтерирования $x$. Такая зависимость, например, была получена экспериментально для близкого по параметрам соединения гидрида титана в [21]. К сожалению, экспериментальных данных по величине удельной эрозии для $\mathrm{ZrD}_{0.67}$ мы на сего- 
дняшний день не имеем. Однако преимущество данного метода заключается еще и в том, что все необходимые параметры для оценки композиционного состава плазмы КП этим способом (в том числе и значение удельной эрозии $g$ ) могут быть измерены экспериментально для реальных электродных материалов и параметров дугового разряда. Таким образом, конвективный характер десорбции дейтерия при образовании кратера в ячейке КП приводит к тому, что отношение количества ионов дейтерия к количеству ионов циркония в плазме КП в 7-10 раз превосходит эту величину в катоде.

Фаза диффузионного восстановления однородной концентрации в катоде длительностью $t_{\text {res }}$ начинается после того, как температура поверхности катода станет меньше $\sim 1100 \mathrm{~K}$. При этом результаты моделирования позволяют говорить о том, что за время остывания катода до комнатной температуры ( $2 \mu \mathrm{s})$ восстановление концентрации дейтерия в вале кратера не существенно. Так что фаза диффузионного восстановления происходит при температурах, близких к комнатной $(\sim 300-400 \mathrm{~K})$. При данной темпеpaтуре $D_{D} \sim 10^{-10}-10^{-9} \mathrm{~cm}^{2} / \mathrm{s}[18,19]$ и для высоты вала $h_{w} \sim 0.2-0.3 \mu \mathrm{m}$ длительность фазы восстановления составляет $t_{\text {res }} \approx h_{w}^{2} / D_{D}(300 \mathrm{~K}) \sim 1-10 \mathrm{~s}$. Так как $t_{\text {res }}$ много больше времени жизни ячейки $t_{c}$ и даже много больше длительности дуги в наших экспериментах, увеличение длительности разряда должно приводить к функционированию новых ячеек КП на уже эродированных и соответственно частично десорбированных участках катода. Это обстоятельство может являться качественным объяснением уменьшения доли ионов дейтерия при увеличении длительности дуги, которая наблюдалась нами экспериментально. Однако для получения количественных оценок данного эффекта результатов рассматриваемой модели не достаточно. Необходимо более детально исследовать характеристики перемещения всего катодного пятна в течение импульса разряда в реальных экспериментальных условиях.

\section{Заключение}

На основании проведенного анализа можно сформулировать следующую интерпретацию результатов экспериментов, которые приведены на рис. 3. При малых токах дуги функционируют в основном пятна первого типа, для которых характерны низкие токи и малые времена функционирования. Эрозионные процессы в этом случае носят взрывной характер [15], поэтому содержание ионов различных элементов в плазме дуги близко к их атомарному содержанию в катоде. В наших предыдущих экспериментах $[2,3]$ зафиксированный рост ионов дейтерия в плазме в начальной стадии токового импульса был связан с задержкой прихода ионов циркония к границе эмиссии (рис. 2). С ростом тока по мере перехода к пятнам второго типа основную роль начинают играть рассмотренные выше процессы десорбции дейтерия при функционировании ячейки катодного пятна второго типа и доля ионов дейтерия возрастает до значений порядка $80 \%$ и выше.

Снижение доли ионов дейтерия в плазме дуги при увеличении длительности импульса обусловлено, на наш взгляд, уменьшением содержания дейтерия на разогретых участках катода, где происходит инициирование новых катодных пятен. Для хорошо очищенных катодов инициирование новых катодных пятен происходит в основном на бруствере кратера (см., например, [22]), образованном вытеснением жидкометаллической фракции при функционировании старого катодного пятна. Как показали наши расчеты, эта фракция практически не содержит дейтерия из-за его термической десорбции.

Кроме этого, важную роль может играть локальный нагрев участка катода, на котором происходит функционирование катодных пятен. Ввиду хаотического движения катодные пятна не обладают „памятью“ [23], т.е. могут неоднократно инициироваться в тех же местах. Для оценки температуры в зоне их функционирования $S_{c s}$ можно записать

$$
T-T_{0} \approx \frac{k I_{a} U_{a}}{\rho c_{p} S_{c s}} \sqrt{\frac{t}{a}},
$$

где $k$ - доля энергии вакуумной дуги, выделяемой в катоде, $I_{a}$ и $U_{a}$ - ток и напряжение горения дуги, $\rho, c_{p}$ и $a$ - плотность, теплоемкость и температуропроводность. Для тока в $1 \mathrm{kA}$ и длительности импульса $10 \mu \mathrm{s}$ существенный рост температуры $\Delta T>500 \mathrm{~K}$ возможен при $S_{c s}<0.1 \mathrm{~cm}^{2}$ (при оценке использовались значения теплофизических характеристик чистого циркония, параметр $k$ был равен 0.3 [24]).

Точно оценить величину $S_{c s}$ сложно, однако в пользу того, что эта величина действительно может быть меньше $0.1 \mathrm{~cm}^{2}$, говорит тот факт, что при хаотическом движении средний квадрат смещения катодного пятна $R^{2} \approx D t[25]$, где типичная величина параметра диффузии для металлов $D \approx 10 \mathrm{~cm}^{2} / \mathrm{s}$, поэтому $R^{2} \approx$ $\approx 10^{-4} \mathrm{~cm}^{2}$, т.е. на площади $0.1 \mathrm{~cm}^{2}$ укладываются траектории нескольких сотен независимых катодных пятен при длительности импульса $10 \mu \mathrm{s}$. Очевидно, что увеличение длительности импульса горения дуги ведет к большим потерям дейтерия на разогретой части катода из-за термической десорбции, и, как следствие, меньшему содержанию его ионов в дуговой плазме. К аналогичному результату должен приводить и рост энерговклада в катод. Однако ситуация здесь не столь однозначная из-за увеличения количества одновременно функционирующих катодных пятен при росте тока. Известно [21], что на катодах, допированных водородом, уменьшается средний ток на катодное пятно и средний размер кратера. Специально проведенные эксперименты показали, что для дуги с длительностью импульса $4 \mu \mathrm{s}$ при повышении амплитуды тока с 1.2 до $2.5 \mathrm{kA}$ доля ионов дейтерия в плазме снизилась с 83 до 75\%, а для длительности импульса $17 \mu$ s при увеличении тока с 600 А до $1.5 \mathrm{kA}$ - с 62 до $50 \%$.

Работа поддержана грантом РНФ № 14-19-00083. 


\section{Список литературы}

[1] Гулько В.М., Ключников А.А., Коломиеи М.Ф., Михайлов Л.В., Шиканов А.Е. Ионно-вакуумные приборы для генерации нейтронов в электронной технике. Киев: Техника, 1988. 136 с.

[2] Баренгольи, С.А., Карнаухов Д.Ю., Николаев А.Г., Окс Е.М., Савкин К.П., Уйманов И.В., Фролова В.П., Шмелев Д.Л., Юиков Г.Ю. // ЖТФ. 2015. Т. 85. Вып. 7. C. $45-54$.

[3] Юиков Г.Ю., Николаев А.Г., Фролова В.П., Окс Е.М., Румянщев Г.С., Баренгольи, С.А. // Письма в ЖТФ. 2014. Т. 40. Вып. 23. С. 74-81.

[4] Yushkov G.Y., Nikolaev A.G., Oks E.M., Frolova V.P. // Rev. Sci. Instrum. 2016. Vol. 87. N 2. P. 02A905.

[5] Nikolaev A.G., Oks E.M., Savkin K.P., Yushkov G.Yu., Brown I.G. // Rev. Sci. Instrum. 2012. Vol. 83. N 2. P. 02A501.

[6] Bugaev A.S., Vizir A.V., Gushenets V.I. et al. // Laser Part. Beams. 2003. Vol. 21. N 2. P. 139-156.

[7] Yushkov G., Oks E., Anders A., Brown I. // J. Appl. Phys. 2000. Vol. 88. N 10. P. 5618-5625.

[8] Кирьянов Г.И. Генераторы быстрых нейтронов. М.: Энергоатомиздат, 1990. $224 \mathrm{c.}$

[9] Суховеев С.И. // ЖТФ. 1979. Т. 49. Вып. 5. С. 1027-1029.

[10] Jutner B., Puchkarev V., Hantzsche E., Beilis I. Cathode Spots, Handbook of Vacuum Arc Science and Technology. 1995. P. 124.

[11] Cuneo M.E. // IEEE Trans. Dielectr. Electr. Insul. 1999. Vol. 6. P. $469-485$.

[12] Scott C.D., Lefebvre M. // J. Thermophys. Heat Tr. 1996. Vol. 10. P. 426-435.

[13] Шмелев Д.Л., Баренгольи, С.А., Щитов Н.Н. // Письма в ЖТФ. 2014. Т. 40. Вып. 18. С. 16-23.

[14] Кесаев И.Г. Катодные процессы электрической дуги. М.: Наука, 1968. 244 с.

[15] Месяи Г.А. Эктоны в вакуумном разряде: пробой, искра, дуга. М.: Наука, 2000. 424 с.

[16] Uimanov I.V., Shmelev D.L., Barengolts S.A. // Proc. 27th Int. Symp. on Discharges and Electrical Insulation in Vacuum. 2016. Vol. 1. P. 357-360.

[17] Месяи Г.А., Уйманов И.В. // Известия вузов. Физика. 2015. Т. 58. Вып. 9/2. С. 204-208.

[18] Gelezunas V.L., Conn P.K., Price R.H. // J. Electrochem. Soc. 1963. Vol. 110. N 7. P. 799-805.

[19] Гидриды металлов / Под ред. В. Мюллера, Д. Блэкледжера, Дж. Либовица. Пер. с англ. М.: Атомиздат, 1973. $432 \mathrm{c}$.

[20] Физические величины. Справочник / Под ред. И.С. Григорьева, Е.З. Мейлихова. М.: Энергоатомиздат, 1991. 1232 с.

[21] Shkol'nik S.M. // IEEE Trans. Plasma Sci. 2001. Vol. 29. P. 675-683.

[22] Juttner B., Kleberg I. // J. Phys. D: Appl. Phys. 2000. Vol. 33. P. 2025-2036.

[23] Anders A. Cathodic Arcs. NY.: Springer, 2008. 540 p.

[24] Daalder J.E. // J. Phys. D: Appl. Phys. 1977. Vol. 10. N 16. P. 2225-2234.

[25] Jüttner B. // J. Phys. D: Appl. Phys. 2001. Vol. 34. N 17. P. R103-R123. 